31 March 04

\title{
Enrico Fermi and the Miracle of the Two Tables
}

\author{
by Gerald Holton
}

There is something quite special about the place of Fermi in history. We all know that in the turbulent flow of time there have arisen, on rare occasions, events that did not fit any previously made plan, but which nevertheless powerfully shaped all subsequent history. Among the most spectacular examples is of course the discovery by a captain, born in Genoa, who set sail toward Asia, but serendipitously encountered instead the land that came to be called the New World. From that moment, the clock for the modern period was set. Another instance of a similar sort was when a then still obscure professor of mathematics at the University of Padua, having used his homemade spyglass for terrestrial explorations, raised it to scan the heavens, and was the first to see there the evidence, in the appearance of the Moon, Jupiter, and Venus, that the existing worldview had to be replaced by a new one. That is when the clock for modern science suddenly came alive. And a third example was a seemingly unplanned event that took place in Rome in October 1934, with transforming consequence--for large sections of physics, chemistry, engineering, medical research, and ultimately for politics and warfare.

I refer of course to the discovery, in October 1934, by Enrico Fermi and members of his group, of what was later called the "moderator effect," the way to turn fast neutrons into slow ones, and the startling new phenomena those neutrons could induce. By itself, such matters would be of interest only to fellow physicists, and ultimately to those good people in Stockholm. But about four years after Fermi's discovery, in a publication in 
which Lise Meitner and Otto Frisch immediately recognized the evidence for nuclear fission, its authors, Otto Hahn Hahn and Fritz Strassmann, referred to the key role in their work of "slowed-down neutrons." They did not happen to mention the Italians who had found how to make those slow neutrons. But it can be said that on a day in October 1934, the clock began to tick which ever since has marked the nuclear age in world affairs.

\section{I}

Despite their diversities, these examples, and others of this sort throughout history, have in common not only the initial unintention on the part of the discoverers, and the extraordinary transformations they eventually caused. They also are of the rare sort of research findings which do not correspond to the more usual ones, being not merely discoveries of new facts, or verifications of predictions, or answers to old questions, or support to prop up an unstable theory. They are not just the addition of another brick to the ever-unfinished Temple of Isis. Rather, they suddenly open up access to an area beyond the map of established knowledge, thereby permitting an exploration of a new continent of fruitful ignorance. For what is most prized in science is the discovery of a vast absence of knowledge, of a range of hitherto undiscovered truths, owing to the breakdown of a standard model.

Superficially, those examples of profound discoveries might tend to support the view that the course of history itself is decided by the works of great men, to use the title of Wilhelm Ostwald's famous book. That opinion is contrary to the other old illusion, that it is history which shapes the ideas and acts of even the greats. But each of these two 
opinions is itself illusory. Any study of actual cases soon shows that both mechanisms together are constantly at work. As the psychologist Erik Erikson put it, "an individual life is the accidental coincidences of but one life cycle with but one segment of history." Even the most fateful chance observation has its own prehistory; and conversely, there is no proof that even the most turbulent event in world affairs has been caused by some overarching Zeitgeist.

\section{II}

From childhood on and into his early student years, young Fermi was recognized by his teachers, acquaintances and friends as a prodigy. Relying largely on self-study--a mode typical of great scientists, from Kepler to Faraday to Einstein - he soon became precociously at home with modern physics, enjoying equally the experimental and the theoretical sides. As a very young man Fermi turned to quantum theory, probably the first to do so in Italy, where that subject was considered a sort of no-man's land between physics and mathematics, rather than a promising research site. That part of physics was not taught in Italian universities there, and a dissertation in theoretical physics as such would have been shocking.

Therefore, Fermi's dissertation at the University of Pisa (finished at age 21) had to be an experimental one--on images obtained with monochromatic x-rays by means of a curved crystal. To build the necessary apparatus, Fermi persuaded his fellow students, Franco Rasetti and Nello Carrara, to help him—a first hint of his capacity for organizing teams. Typical also of his later years, Fermi was not satisfied with putting in print the experimental findings (in his seventh published paper, dated 1923). Before that he had 
published a separate, lengthy theoretical paper on the properties and theory of x-rays.

There he showed that he commanded the whole literature-including von Laue, Bragg, Moseley, Barkla, Sommerfeld, Maurice de Broglie, Debye, Scherrer, etc.--in all their several languages. And already then he was keeping physics almost constantly in his thoughts. There is a story, perhaps apocryphal but believable, that one of Fermi's friends once found him pacing up and down in a room, with a preoccupied look. Concerned, his friend asked if Fermi was troubled by something. "No," Fermi replied. "I am just estimating by how much I am depressing the wooden floor as I walk along it."

Experimental x-ray studies, and even quantum physics, were by no means the only subjects enchanting the young physicist. It became important for his subsequent career that starting at age 19, Fermi's first five published papers were all on relativity theory. Most of them showed his mastery of the methods of general relativity, the theory just recently confirmed by Eddington's experiment. To be sure, most of the older generation of physicists in Italy were still skeptical and hostile to that theory. But like Wolfgang Pauli and Werner Heisenberg, at about the same time and at the same young age, Fermi had evidently been captivated by Herman Weyl's new book, Raum, Zeit, Materie, for which Einstein himself had written an enthusiastic review in 1918. Fermi contributed to relativity a theorem of permanent value (later called Fermi coordinates), and soon it was incorporated into textbooks on General Relativity. Luckily, Italy had at that time several master mathematicians working in that field, such as Tullio Levi-Civita and Gregorio Ricci-Curbastro. They, and other mathematicians of first rank, including Guido Castelnuovo, Federico Enriques and Vito Volterra, began to notice Fermi's papers, and were ready to support his rise. 
Yet, again contrary to ordinary expectations, Fermi properly soon realized that relativity theory was not the field in which to build his own career. From 1921 to 1925, he had no less than thirty-one publications, as reproduced in his Collected Papers, most in theoretical physics--even though he knew that there was not a single University chair available for it in all of Italy. His wide-ranging interests and sheer productivity were as astonishing as his self-confidence.

Since our theme is the formation of Fermi's team, I can point out that we have now already met the first member of the group that would soon be formed, the enormously talented experimental physicist and Fermi's schoolmate, Franco Rasetti. It is also time to introduce Orso Mario Corbino, a crucial figure in the eventual rise of Fermi and his group. Twenty-five years older than Fermi, he was widely known for his early work in magneto-optics, for which he had been admired by Augusto Righi of Bologna, considered the previous generation's leading physicist in Italy. After Corbino had been called to the University of Rome, his talent as an administrator and unselfish connoisseur of talent quickly led to his becoming Senator of the Kingdom (1920), Minister of Public Instruction (1921) and Minister of National Economics (appointed in 1923, by Mussolini, although never being a member of the Fascist Party).

Corbino's keen scientific mind, combined with his hope to put Italy again on the map as a center of great physics research, led him to mourn the sorry state of physics there, symbolized for him by Righi's death in 1920 . He saw clearly that Italy was then unable to take advantage of the world-wide rise of opportunities in the new physics of the day. Without realizing it, by 1920 Corbino was ready to discover a Fermi-just as 
Enrico Fermi, for his part, must have known that without the help of such a man there might never be a Fermi group.

After Fermi's graduation from Pisa, he returned to Rome in 1921, at age 20, living with his parents and his older sister, as he was to do for several more years-- a member of a closely knit family. At the moment, he had neither a job nor prospects for one. But one day he decided to make a first visit to Corbino. The two men immediately took to each other. With Corbino's help, Fermi obtained fellowships, spending unhappy months at Göttingen, and happy ones in Leyden under Paul Ehrenfest, then a couple of years in temporary posts at Florence, working with Rasetti. At last, in 1926, Fermi was appointed to the new Chair of Theoretical Physics at the University of Rome, a move engineered by Corbino who was officially the director of the University's Physical Institute at Via Panisperna 89a, the building in which the top floor was in fact the flat of Corbino's family.

III

Now Fermi could begin to put his and Corbino's dream into reality. But that was not going to be easy. So far, Fermi had admirers, but no followers. The outlook for building a school of bright young physicists was still dark. There was not even an Italian text on atomic physics for advanced university students, and of those students there were only a handful, since the expectation for eventual university employment was extremely poor. Fast action was called for. First, Fermi wrote and published in 1927 that missing textbook. Corbino used his influence to bring Franco Rasetti from Florence to Rome, eventually settling him into a professorship for spectroscopy, created for that purpose. 
(How we all would have liked to have had a Corbino on our side! He seems the ideal candidate as patron saint for bright young scientists.) And now Fermi and Rasetti began to recruit promising university science students for their Istituto.

Emilio Segrè, a student in engineering in Rome, met Fermi, who was only four years older, with the result that Segrè knew instantly that here was an extraordinary teacher, scientist and human being. That autumn, with Corbino smoothing the administrative problems, Segrè transferred his studies to the physics section of the University, thereby becoming Fermi's first pupil. In Segrè's words, "The Roman School had started." Segrè in turn persuaded his friend Ettore Majorana to join the group, at least informally.

Here an important aside is called for. The last paragraph above contain several clues to the vitality and unique characteristics of the formation of the Roman School. First, Corbino was ever ready to help, in any way. Second, all of "Corbino's boys," as they came to be called later, were within a few years of the same age. Third, among them there was a camaraderie in which the only trace of hierarchy was the acknowledged centrality of Fermi's brilliance. Finally, almost all members of the group were part of one social network. They typically even spent parts of their vacations together at the seaside or in the mountains. For example, in the summer of 1925, Fermi was in the mountains with the families of Levi-Civita, Castelnuovo, and Ugo Amaldi. Amaldi's seventeenyear-old son, Edoardo, was fascinated by the scientific talk, and ended up accompanying Fermi on a bicycle tour of the Dolomites. A bonding had begun there which, together with Corbino recruiting him from the engineering class, resulted two years later in Edoardo becoming part of Fermi's physics group at the Institute. 
If all this sounds a bit like the behavior of a stereotypical Italian family, let us remember that this was not the way things then generally arranged themselves in physics laboratories in, say, Göttingen, or, for that matter, in New England. At any rate, we see that a critical mass was being formed at the Institute in Rome. The group's younger students became more and more competent, partly through participating in experiments with Rasetti, but above all through Fermi's constant care and the value of his informal theoretical seminar. For Fermi was an ideal teacher-with one occasional exception: as John Marshall later recalled, Fermi sometimes saw to it that he was the only person near the blackboard who had the chalk. Marshall added: "It was very difficult to argue with the only person who had the chalk."

Fermi's typical mode of teaching was to keep things clear and seemingly improvised. He distrusted abstract theories such as the quasi-philosophical Copenhagen versions of quantum mechanics, favoring instead the visualizable approach of Schrödinger. Hans Bethe referred admiringly to Fermi's way as "enlightened simplicity."

Fermi thought and taught about physical phenomena in a unique way. Just as his experimental equipment functioned well despite being often assembled out of cannibalized pieces and in the least complex manner, so also did Fermi consider Nature herself put together in the most parsimonious way. That is to say, he recognized again and again the same pattern to be at work in completely different contexts. Thus he applied the same idea of scattering length, first to explain the pressure shift of spectral lines (Document 95 in Fermi's Collected Papers), and second to understand artificial radioactivity produced by neutron bombardment (Document 107) — even using the same diagrams in the publications. Or again, he applied the same statistical theme to atoms on 
the one hand, and to neutrons on the other. Fermi's great paper on beta decay, at its core, treats the emission of electrons and neutrinos in nuclear events as analogous to the emission of photons from atoms in excited states (Document $80 \mathrm{~b}$ ). As Fermi's colleague at the University of Chicago (and co-author on two papers), the astrophysicist Subrahmanyan Chandrasekhar put it (Fermi Collected Papers, v. II, p. 923): "The motions of interstellar clouds with magnetic lines of force threading through them reminded him of the vibrations of a crystal lattice; and the gravitational instability of a spiral arm of a galaxy suggested to him the instability of a plasma and led him to consider its stabilization by an axial magnetic field."

One recognizes here the way a thematic presupposition guides some scientists' understanding of how Nature works at the fundamental level. Einstein's basic assumption was again and again that entirely different phenomena are aspects of one grand unity. Niels Bohr, quoting a saying of Friedrich Schiller, thought that truths may be found best "in the abyss" between contrary theories. Fermi thought of each phenomenon as exhibiting one of only a relatively small number of different basic scenarios of which Nature availed herself; and of these, Fermi kept a catalogue throughout his life.

\section{IV}

In order to learn new skills, members of Fermi's group, already international in outlook, visited laboratories abroad. Rasetti travelled to Millikan in Pasadena and later to Lise Meitner in Berlin. Segrè went to Pieter Zeemann in Amsterdam and Otto Stern in Hamburg. In turn, more students from other universities transferred to join the Rome group, including Eugenio Fubini, Ugo Fano, and Bruno Pontecorvo. They were attracted 
by Fermi's work, for example on the quantum theory of radiation, on statistics, above all on the theory of beta decay, the paper first published in 1933, after having been rejected by the editor of the journal Nature as "containing abstract speculations too remote from physical reality." Also, a good number of young physicists came from abroad, to visit and sometimes to stay for longer periods and collaborate. They included Hans Bethe, George Placzek, Felix Bloch, Rudolf Peierls, Fritz London, Edward Teller, Eugene Feenberg. And before that, there were collaboration with and visits from colleagues at other Italian universities, such as Renato Einaudi from Turin, but perhaps most frequently from the newly flourishing physics group in Florence under Antonio Garbasso, including Bruno Rossi, Gilberto Bernardini, Giuseppe Occhialini, Perisco, Giulio Racah, and Sergio De Benedetti.

But what would be the freshly hatched young Roman group's contribution to physics? Up to 1929, the largest part of their teamwork was still in spectroscopy. But from then on, it became clear that remarkable changes in physics abroad required a new direction if Italian physics was to reach world-class level. The historical development of physics itself revealed to the Fermi team what these young men had been preparing themselves for, in all those years of wide-ranging study and perfection of various skills. The quantum mechanics of Bohr, Heisenberg, Pauli, Dirac, Schrödinger was taking center stage in the field of theory; and on the experimental side, nuclear physics was being transformed in exciting ways, by the findings of Chadwick, Urey, Davisson and Germer, Carl Anderson, Nedermeyer and Street. The proton-neutron model of the nucleus was becoming plausible. The neutrino hypothesis of Pauli was tantalizing; and E. O. Lawrence's cyclotron was a much-envied sensation. 
In a speech in September 1929, Corbino showed he had already smelled out that nuclear physics was, in his words, "the true field for physics of the future." Now, the core members of the team, who had patiently and often at great personal cost stuck together for years, re-educated themselves in systematic study. Amaldi led a special seminar on radioactivity, and the group learned how to build neutron sources, construct a cloud chamber, and make Geiger counters. Some additional research funds were obtained from the Italian National Research Council (CNR). And all this was being done without the group realizing precisely what and when an opportunity would come along for using that new knowledge, to achieve the ultimate desire of the team: to make, at long last, a world-class discovery. It was a curious moment in the history of science: Arguably the first modern research team in physics, it was waiting for something to happen that could put the group to use for a high purpose.

\section{V}

Remarkably soon, something did happen, entirely out of the blue. In Paris, the French physicist Irène Curie Joliot and her husband Frédéric Joliot, had been using alpha articles from polonium, sent into a cloud chamber to bombard aluminum, so causing the immediate emission of positrons from the target. One day, in January 1934, Joliot noticed by chance that the emission of positrons persisted when the polonium source was taken away. A new radioactive isotope had been created by the alpha particle bombardment. One might add here that earlier, a non-discovery of artificial radioactivity took place in E. O. Lawrence's cyclotron laboratory in Berkeley. As Lawrence confessed in his Nobel Prize speech (for 1939, but given in 1951), "Looking back, it is remarkable 
that we [at Berkeley] managed to avoid the discovery," by neglecting the fact that the Geiger counters continued their chatter even after the 27 -inch cyclotron had been turned off.

The Joliot-Curies' discovery opened a window on a new landscape of exciting ignorance. While others immediately rushed to explore this territory, using alpha particle sources, Fermi made the crucial decision to see if a beam of neutrons would also produce artificial radioactivity. It seemed to him reasonable to expect that the lack of charge of neutrons, many of them emerging at high energies from the radon-beryllium sources would permit a great effect on the charged nuclei of the targets, despite the admittedly still relative weakness of the sources available to them.

On March 25, 1934, Fermi was able to publish the first results of the group's experiments, in the journal of the National Research Council, Ricerca Scientifica. It was the first of ten such publications in the spring of 1934, sometimes one appearing each week. From the third to the tenth of these publications, the list of authors was always given as follows: "E. Amaldi, O[scar] D'Agostino [a young chemist who happened to come back to Rome on a vacation from a fellowship in Paris, but happily was pressed into service], E. Fermi, F. Rasetti, E. Segrè." Note that all core members of the "family" were listed in alphabetical order, and that, perhaps for the first time in the whole physics literature, there were as many as five accredited authors.

Fermi typically had decided to test all available chemical elements for artificial radioactivity, going methodically up the periodic table. The team divided the labor in a cooperative way--getting the targets, monitoring the electric circuitry of the Geiger counters, making chemical analyses, etc. Fermi's group, from the beginning, generally 
tended to work together on one project - unlike the operation at, say, Rutherford's own Cavendish Laboratory, where different small groups worked on different projects whose commonality was chiefly that they represented different parts of Rutherford's wideranging interests.

The work in Rome was now quite frantic and exhausting for some months, and a few mistakes were made. None was later regretted by Fermi's group more than the presumed identification of transuranium radioactive products, produced by irradiating the elements thorium and uranium with neutrons. The same mistake was made by others at the same time, including the Joliot-Curies. One is tempted to be thankful for that, because otherwise fission might have been discovered earlier, when only two countries might have been interested in putting it to use in a weapon-Germany and the Soviet Union. At any rate, altogether the Roman team irradiated over sixty elements with neutrons, producing by artificial radioactivity forty-four new isotopes. Here was excellent work, the unexpected first fruits after the long wait and preparation. The Fermi group was now widely noted. Because at the time such publications had to be by law first in Italian, I. I. Rabi at Columbia University is said to have advised, "Well, now we all have to learn Italian." Rabi also began to consider the prospect of Fermi joining the physics faculty at Columbia_ - as was eventually to happen, with spectacular results.

VI

Meanwhile, without realizing they were now standing at the threshold of a startling discovery, with its long resonance into science and world history, the whole Roman group took a break for the lengthy summer vacation, getting away from the heat 
of Rome. When they reassembled at the Istituto in the fall of 1934, they were joined by Bruno Pontecorvo, a close family friend of Rasetti. But by mid-October things began to go wrong. Their whole experimental activity was upset by a strange inconsistency in the results of irradiation of targets during an attempt to calibrate the degrees of induced radioactivity. The readings turned out to depend on the tables used as support of the equipment. One table, once the bearer of the spectroscopes of earlier days, was made of wood; the other, not far away, was a shelf made of stone. When an experiment on inducing radioactivity, in a target made of silver, was made on the wooden table, a markedly greater activity resulted than if the same experiment was tried on the stone support. The group christened it the "miracle of the two tables."

To get to the bottom of this puzzle, Fermi initiated a systematic observation, starting October 18, 1934. He and his team reasoned that perhaps the lead housing around the target affected the neutrons reaching the target in those two cases, and they observed that the interposition of a block of lead changed the activation somewhat. Therefore, Fermi decided to make a lead filter, a wedge of varying thickness, to insert into the neutron beam. As Segrè put it later, on that day, "Persico and Bruno Ross [were] there on a visit, kibitzing." The account of the events on that morning was later told by Fermi to Subrahmanyan Chandrasekhar, who published his report (Fermi, Collected Papers, v. 2, p. 927). The essential last paragraph was repeated verbatim by others close to Fermi, such as Edoardo Amaldi and Emilio Segrè ${ }^{\mathbf{1}}$.

"I will tell you how I came to make the discovery which I suppose is the most important one I have made. We were working very hard on the neutron-induced

\footnotetext{
${ }^{1}$ A detailed version of the events was given by Laura Fermi in her book, Atoms in the Family (1954, p. 98). It differs in some details, but comes to the same conclusion.
} 
radioactivity, and the results we were obtaining made no sense. One day, as I came to the laboratory, it occurred to me that I should examine the effect of placing a piece of lead before the incident neutrons. And instead of my usual custom, I took great pains to have the piece of lead precisely machined. I was clearly dissatisfied with something: I tried every 'excuse' to postpone putting the piece of lead in its place. When finally, with some reluctance, I was going to put it in its place, I said to myself: 'No! I do not want this piece of lead here; what I want is a piece of paraffin.' It was just like that: with no advanced warning, no conscious, prior, reasoning. I immediately took some odd piece of paraffin I could put my hands on and placed it where the piece of lead was to have been."

The result was immediately obvious: a great increase in the radioactivity induced in the target, even if the target and the paraffin filter were placed on the stone shelf. As Segrè recalled, at about noon, "everybody was summoned to watch the miraculous effect of the filtration by paraffin." And in a "still extremely puzzled" state, "we went home for lunch and our usual siesta." "When we came back at about three in the afternoon, Fermi had found the explanation of the strange behavior of filtered neutrons. He hypothesized that neutrons could be slowed down by elastic collisions, and in this way become more effective - an idea that was contrary to our expectation" (in Segrè, Enrico Fermi, Physicist, p. 80).

As Amaldi reported, it was only later that the so-called 1/v law was determined, i.e., that the capture cross-section $\left(\sigma_{c}\right)$ was inversely proportional to the speed of the neutrons at low velocities. But on that day, the miracle of the two tables was unmasked. Fermi realized that the hydrogen nuclei in the wooden table had greatly slowed some of the incident neutrons, being of about the same mass, and then had scattered them to the 
target, producing the unexpected effect on it, whereas the heavy nuclei in the stone table could slow and scatter neutrons only very poorly. Repeating the experiment quickly by using water instead of paraffin helped prove Fermi's initial hypothesis. Moreover, the enhanced radioactivity was also observed for copper, iodine, and aluminium.

That evening, in Amaldi's home, they all met to prepare a short report of their work for the Ricerca Scientifica, with Fermi dictating, Segrè writing, Rasetti, Amaldi and Pontecorvo excitedly adding their comments. Amaldi's wife Ginestra, who was working with that journal, saw to it that the article would be published within two weeks, with preprints - another novelty--becoming available within days, and sent out to some forty of the most prominent researchers in the field. Soon the whole profession knew that the Roman group had crossed a new frontier. It was a climactic moment for Fermi's team in Rome, and--as it turned out--for the world on its path into the uncertain future.

\section{VII}

But in that story so far, there is a haunting puzzle. Fermi was the most rational and least impulsive of scientists; yet, not by accident or chance but by sudden, determined action, "with no advanced warning, no conscious, prior reasoning," he had placed the crucial piece of paraffin before the neutron source. ${ }^{2}$ This seems to me an example of a kind of intuitive intelligence which sometimes guides brilliant minds in the

\footnotetext{
${ }^{2}$ One historian of science was so astonished by the report of an action so uncharacteristic of Fermi that he even doubted the account reported by Chandrasekhar. But that idea must surely be dismissed. Chandrasekhar, who was one of the most distinguished and precision-minded scientists, even felt it necessary to start his report with a footnote: "His [Fermi's] account made so great an impression on me that though this is written from memory, I believe that it is very nearly a true verbatim account." Moreover, he published it (in 1965) when those who had "kibbitzed" that morning in 1934,--Rossi and Persico-were still alive; and as mentioned, Fermi's co-workers, Segrè and Amaldo, endorsed Chandrasekhar's account as given above, quoting it in full.
} 
early phases of their research. That concept is now rarely mentioned, least of all by scientists themselves, who tend to shy away from such a difficult-to-define notions. But it had figured prominently in the writings of philosophers such as Baruch Spinoza, Immanuel Kant, and Henri Bergson. Arthur Schopenhauer, widely read at the time, even held that intuition is the hallmark of genius. Einstein referred to it as "Fingerspitzengefühl," a feeling at the tips of one's fingers, and considered it essential to scientific inquiry (e.g., in his essay, "Motive des Forschens,"1918): "There is no logical path to the elementary laws, but only intuition, resting on empathy gained by experience." Henri Poincaré (in Science and Method, Book II, Chapter 2) noted that it is by logic that we prove, but by intuition that we discover. The scientist and philosopher Michael Polanyi wrote at length about what he called the scientist's "tacit knowledge," largely resulting from one's lengthy immersion or "in-dwelling" in the subject of research. He summarized the result in the simple sentence: "We know more than we can tell." Much earlier, Hans Christian Oersted provided for this rare gift the happy term "anticipatory consonance with nature." And a chemist and great writer, Primo Levi, wrote, "I know with my hands and my nose, with my senses" (in The Voice of Memory, p. 8).

So one might understand that when Fermi's hand was reaching for the "odd piece of paraffin" instead of the lead wedge, he was guided at that moment by a speculation below the level of consciousness, a result of an intimate knowledge of neutron physics, one built up during his years of intense study, discussions and experimentation with 
neutronics. As Dr. Alberto De Gregorio ${ }^{3}$ has shown, Fermi may well have read publications in 1932-33 in which slow neutrons and effects of neutrons on hydrogenous substances were discussed, and he also had participated in the 1933 Solvay Conference which included discussions of these topics. But it is significant that nobody other than Fermi and his group entered into the crash program of producing artificial radioactivity, first with fast neutrons, and then with slow ones, when Fermi was able to draw on resources that had slipped below the conscious level.

In fact, Chandrasekhar's account, given above, is part of a longer piece of his, which reveals that his whole discussion with Fermi had begun precisely with a consideration of the role of "subconscious" ideas in creative work in science: Chandrasekhar wrote (p. 926, Fermi's Collected Papers):

"I described to Fermi [Jacques] Hadamard's thesis regarding the psychology of invention in mathematics, namely, how one must distinguish four different stages: a period of conscious effort, a period of 'incubation' when various combinations are made in the subconscious mind, the moment of 'revelation' when the 'right combination' (made in the subconscious) emerges into the conscious, and finally the stage of further conscious effort. I then asked Fermi if the process of discovery in physics had any similarity. Fermi volunteered and said [there followed his account, as given above]."

There are also other accounts of Fermi's ability to dredge up, from hidden resources, answers to questions facing him. Herbert Anderson recalled that at a crucial moment during the difficult early work in 1939 at Columbia University on the possibility

\footnotetext{
3 "Chance and Necessity in Fermi's Discovery of the Properties of Slow Neutrons," Giornale di Fisica, v. 42, 4 (2001), 195-208, and in a personal communication of an unpublished manuscript, for which I am grateful.
} 
of a chain reaction, under Fermi's direction, "Fermi asked to be left alone for 20 minutes," after which he emerged with a rough estimate of the effect of resonance absorption by uranium. Anderson reported that the estimate, which proved to be correct, "was largely intuitive. Fermi was never far wrong in such things....". One can imagine the positive effect such talent had on Fermi's collaborators. Elsewhere Fermi was even credited with helping reactor engineers to obtain a rough estimate of data not yet measured, such as nuclear cross-section. They did it reportedly by watching Fermi closely for an "involuntary twinkle in his eyes" while reciting to him possible crosssection values. Indeed, the speech by Hans Pleijel of the Swedish Academy at the awarding of the Nobel Prize to Fermi, in December 1938, deployed that key word. Pleijel told his audience: “Along with Fermi’s significant discoveries, and to a certain extent equivalent, can be placed his experimental skill, his brilliant inventiveness, and his intuition...." Fermi's collaborators often said he worked with 'CIF', an acronym for con intuito formidabile."

Science historians have struggled to understand the mechanism behind such examples of "anticipatory consonance with nature." It seems to me another case of finding ourselves before an ocean of fruitful ignorance.

VIII

Not long after the triumph of Fermi's group in Rome, there began a more and more rapid disintegration and near destruction of the Roman group--to use Edoardo Amaldi's phrase - experienced also by the research groups in Florence and elsewhere in Italy. In the early days of Fermi's ascent, some scientists had been helped at least 
indirectly--in hastening appointments and in the availability of some funds - by the Fascist government's attempt to revive the idea of Rome as the center of Western civilization. Among the sixteen institutions founded after Mussolini's assumption of dictatorship in 1925, two were the new Royal Academy of Italy and the National Council of Research (CNR). Fermi's work had required financial support from both, although he himself was by nature and design apolitical, and felt repugnance from the regime's ideology. In turn, the government expressed annoyance with Fermi for his refusing the prestigious chair in physics left by Schrödinger in Zurich, where Fermi, acting as a proxy for Italy's science, would have been highly visible throughout the European continent. The State's police also kept a constant eye on Fermi, as the file still in the Italian State Archive shows.

From the mid-1930s on, a whole set of institutions founded by the Fascist government withered, part of the steady erosion of civilized life. The physicists at the Institute in Rome submerged themselves in hard work, hoping to use physics as "soma," on the model of Aldous Huxley's victims in his novel Brave New World. As their state lurched toward the Ethiopian campaign that started in October 1935, the economic and political dependence of Italy on Nazi Germany grew greatly. In July 1938 came the institution of racist laws in Italy, roughly along the German model. Most of the group's members emigrated. Fermi and his family left Italy in December 1938, bound first for Stockholm, then for the U.S.A. During World War II the authorities in Italy caused the arrest, deportation and death of persons close to the Roman group, including Laura Fermi's father, Augusto Capon. Among the victims sent to the death camps were several relatives of Segrè. 
So few were the years between Fermi's launching the spectacular recovery of Italy's place in physics in the mid-1920s, and its descent by the end of the 30s in horror and flames. Indeed, that whole arc of the brave rise of Enrico Fermi and his group from bleak beginnings, their hard-won achievements, and then the ghastly dissolution forced on the group may well take its place among the symbols of the best and the worst in the history of the twentieth century.

\section{References}

Amaldi, Edoardo, 20 $0^{\text {th }}$-Century Physics. Essays and Recollections: A Selection of Historical Writings by Edoardo Amaldi (Singapore: World Scientific, 1998).

Bonolis Luisa, “Enrico Fermi’s Scientific Work” (in press).

Buck, Barbara, "Italian Physicists and Their Institutions, 1861-1911 (PhD dissertation, Harvard University, 1980.

Fermi, Enrico, Collected Papers, v. I (Chicago and London: University of Chicago Press, 1962).

Fermi, Enrico, Collected Papers, v. II (Chicago and London: University of Chicago Press, 1965).

Fermi, Laura, Atoms in the Family (New York: American Institute of Physics, 1987).

Holton, Gerald, The Scientific Imagination, rev. ed. (Cambridge, MA: Harvard University Press), 1998, Ch. 5.

Pontecorvo, Bruno, Fermi e la fisica moderna (Rome: Editori Riuniti, 1972). 
Proceedings of the International Conference, "Enrico Fermi and the Universe of Physics" (Rome: ENEA, 2003)

Segrè, Emilio, A Mind Always in Motion: The Autobiography of Emilio Segrè (Berkeley, CA: University of California Press, 1993.

Segrè, Emilio, Enrico Fermi, Physicist (Chicago and London: University of Chicago Press, 1970).

Weiner, Charles, ed., History of Twentieth-Century Physics (New York: Academic Press, 1977), especially the essay by E. Amaldi.. 